\title{
Pedunculated liver hemangioma mimicking stomach neoplasm
}

\author{
Sonay Aydın, Erdem Fatihoğlu, Elif Ergün, Pınar Nercis Koşar \\ Department of Radiology, Ankara Training and Research Hospital, Ankara, Turkey
}

\begin{abstract}
Hepatic hemangiomas are the most frequently seen benign liver mass. However, exophytic hepatic hemangiomas, particularly pedunculated ones are very rare. They have various appearances that make the diagnosis difficult. We report a pedunculated hemangioma case which was misdiagnosed as gastrointestinal stromal tumor on computerized tomography scan. Magnetic resonance imaging revealed that the tumor was a hepatic hemangioma with a long peduncle originating from the right edge of the liver, extending into perigastric area. Hepatic hemangioma with a long peduncle can be mistaken with other abdominal masses. Remembering this entity and knowing its imaging characteristics are the keys to diagnosis.
\end{abstract}

Eur Res J 2018;4(2):118-121

Keywords: Pedunculated hemangioma, liver, gastric mass

\section{Introduction}

Hepatic hemangiomas are the most frequently seen benign liver masses (7-20\%), which are seen more often in woman. They are generally single and located frequently in right lobe especially posterior segment and subcapsular area [1]. They are usually less than $3 \mathrm{~cm}$ in diameter, larger ones $(>4 \mathrm{~cm})$ are named as giant hemangioma [2]. Hepatic hemangiomas are asymptomatic and discovered incidentally. Larger lesions may cause symptoms, like a palpable abdominal mass, pain, hemorrhage, jaundice, nausea/vomiting. Exophytic hepatic hemangiomas, particularly pedunculated ones are very rare [3]. Atypical hemangiomas like pedunculated ones have various appearances that make diagnosis difficult.

\section{Case Presentation}

A 70-year-old man with an incidentally found intra-abdominal mass located adjacent to greater curvature of stomach on abdominal ultrasound was referred to radiology department for abdomino-pelvic computerized tomography scan. He was asymptomatic despite the mass and had no family history of abdominal malignancy. On physical examination, there were no abnormal findings. Portal venous phase computerized tomographic images revealed a $22 \times 26$ $\mathrm{mm}$ exophytic mass located adjacent to greater curvature of the stomach (Figures 1a and 1b), mass was considered as gastrointestinal stromal tumor. Endoscopic examination and magnetic resonance imaging was recommended. In addition, there was another $20 \times 15 \mathrm{~mm}$ mass located at segment 6 of 


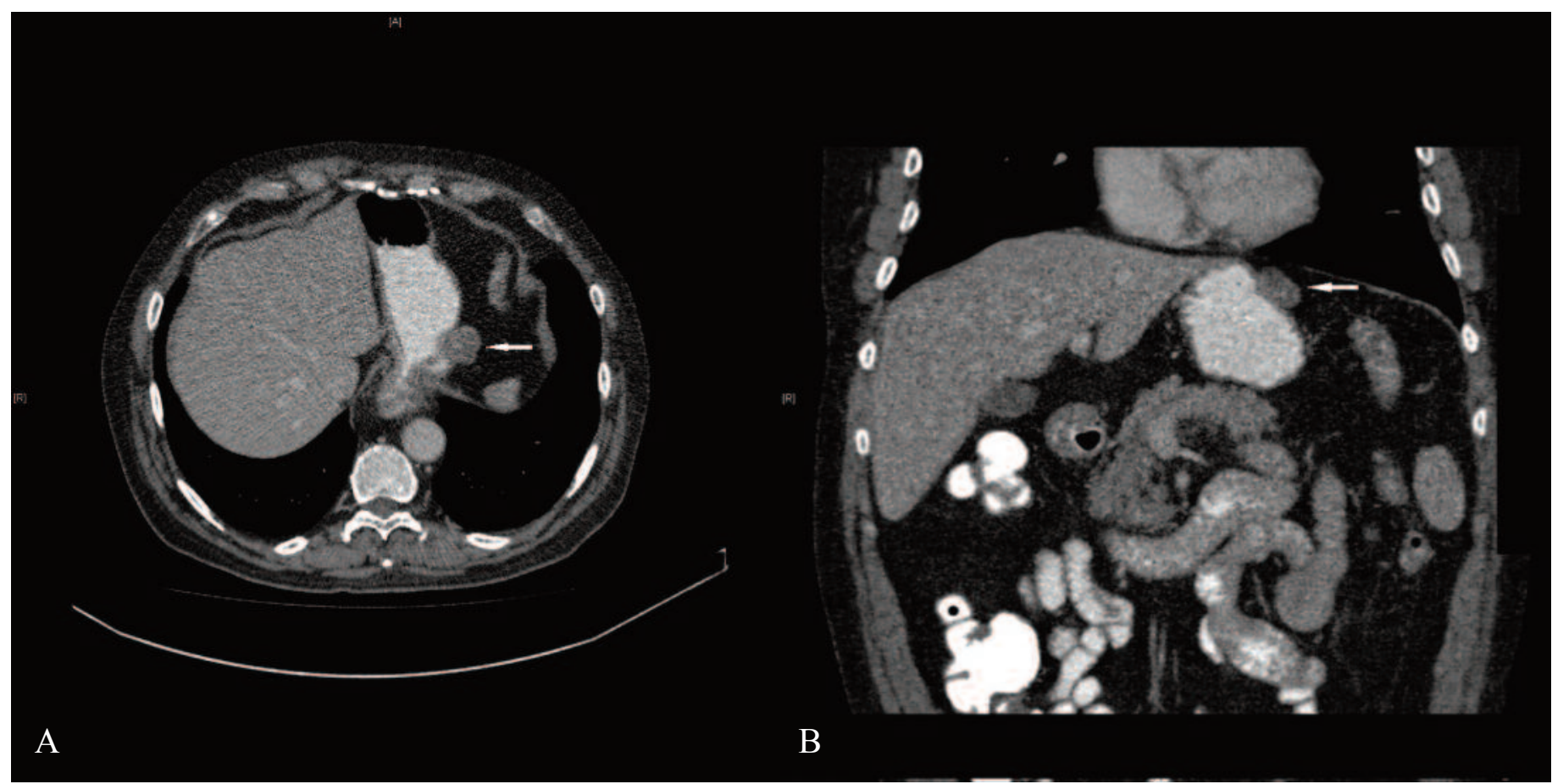

Figure 1. Axial (a) and coronal reformat (b) contrast enhanced CT images showing an exophytic, non enhanced, mass (arrow) located at great curvature of stomach.

liver, with peripheral enhancement. This lesion was diagnosed as hemangioma.

Endoscopic findings were normal. On magnetic resonance imaging, the lesion at segment 6 of liver had peripheral nodular discontinuous enhancement which progressed centripetally on delayed images, consistent with hemangioma. The other lesion, which was considered to be gastrointestinal stromal tumor according to computerized tomography, was originating from left liver lobe and extending exophyticly into perigastric area. It had the same hemodynamic characteristics with the lesion at segment 6 and was seen to be connected to left liver lobe by a peduncle, thus it was described as pedunculated liver hemangioma (Figures $2 \mathrm{a}, 2 \mathrm{~b}, 2 \mathrm{c}$, and 2d).

\section{Discussion}

Hemangiomas are the most common benign tumors of the liver, and the diagnosis of typical hemangioma is easy with the help of various imaging techniques, like ultrasound, computerized tomography or magnetic resonance imaging. However pedunculated hepatic hemangioma is very rare. As far as we know there is 18 reported cases in English literature. Pedunculated hemangioma is known to mimic other abdominal masses, like gastric tumor or other pedunculated hepatic tumors, such as hepatocellular carcinoma, mesenchymal hamartoma, focal nodular hyperplasia, or adenoma. Diagnosis of pedunculated hepatic hemangioma might be difficult as defining the origin of a pedunculated mass is generally problematic [4].

Ultrasound, computerized tomography, magnetic resonance imaging, radionuclide scintigraphy, and angiography are diagnostic methods to identify pedunculated hepatic hemangiomas. Generally, it is difficult to show the origin of the lesion on ultrasound. If the lesion is attached to the liver by a thin pedicle, the peduncle can be nearly undetectable by imaging methods. Multiplane reconstruction computerized tomographic images and coronal or sagittal magnetic resonance imaging may be helpful in diagnosis. On computerized tomography and magnetic resonance imaging, the diagnosis is made by the demonstration of the typical enhancement patterns / signal intensities on both T1- and T2-weighted images [4, 5].

In current case, computerized tomography failed to diagnose the lesion because it was not a dynamic examination. Thus, the typical enhancement pattern for hemangioma could not be revealed by computerized tomography. In addition, the localization of the lesion is atypical for a hepatic mass and the peduncle of the lesion was clearer in magnetic resonance imaging.

Hemangiomas are mostly asymptomatic, but 

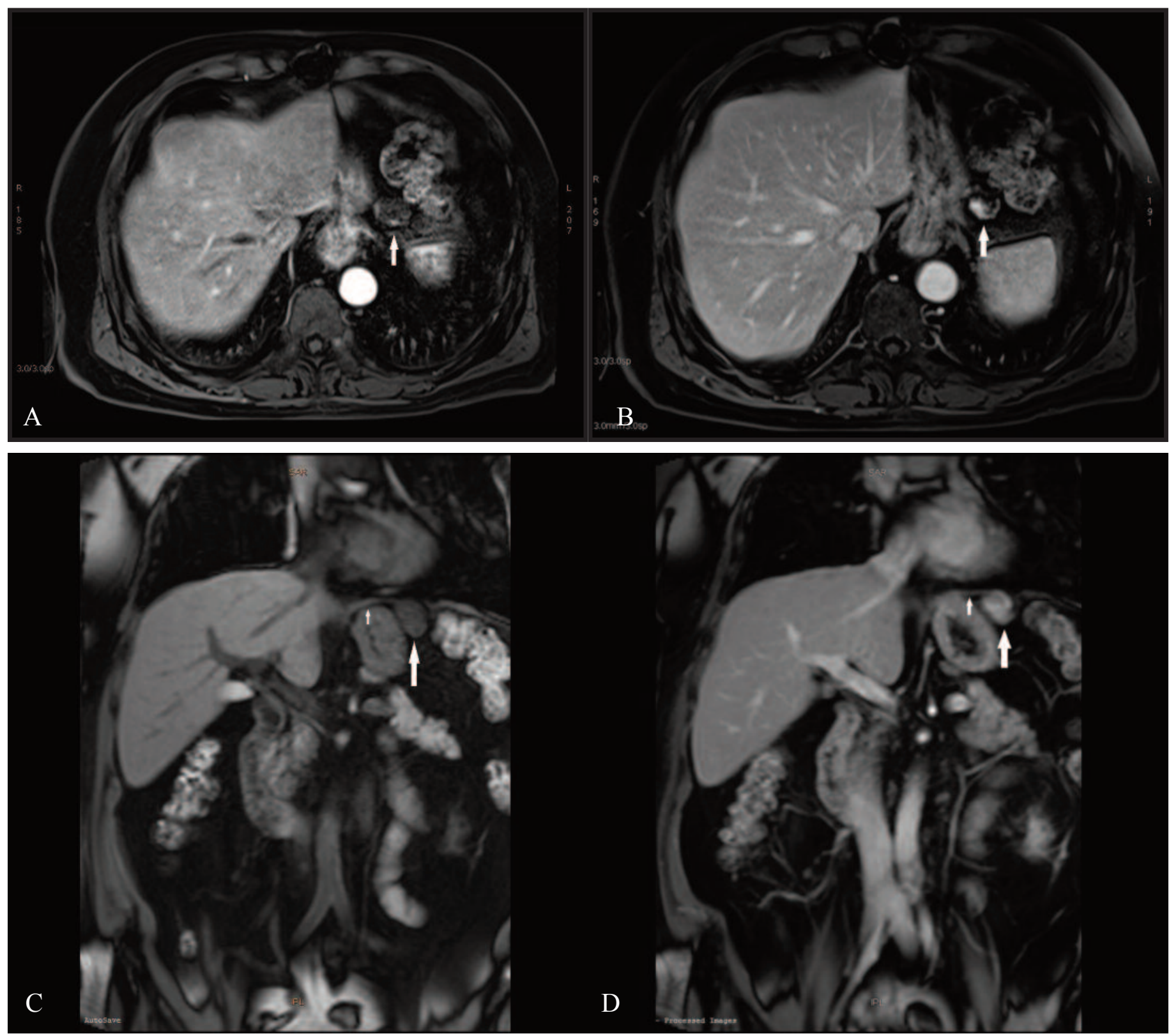

Figure 2. Early arterial (a), portal venous (b) and delayed, contrast enhanced T1 weighted images showing the pedunculated hemangioma (arrow) which has peripheral nodular discontinuous enhancement, progressed centripetally on delayed images. On coronal reformatted non-contrast enhanced (c) and contrast enhanced (d) images, the peduncle of the hemangioma can be seen more easily (peduncle: small arrow, hemangioma: long arrow).

larger ones may produce certain symptoms, including an abdominal mass, pain, nausea, vomiting, jaundice, hemorrhage and sometimes rupture. There is no need for treatment for the majority of hemangiomas, except the ones which present with a palpable mass, pain or complications, as consumptive coagulopathy and rupture can be treated with surgery. Pedunculated hepatic hemangiomas can be complicated by subacute torsion and infarction, and complicated ones must be resected immediately $[3,5]$.

\section{Conclusion}

Pedunculated hepatic hemangiomas uncommon and rare benign liver masses. They show similar imaging features with typical hemangiomas. They should be kept in mind in the differential diagnosis of an upper abdominal mass. Imaging modalities including ultrasound, computerized tomography and magnetic resonance imaging are all capable to diagnose these tumors. Remembering the imaging characteristics and identifying the peduncle are crucial in achieving correct diagnose and avoiding unnecessary diagnostic or therapeutic interventions.

\section{Informed consent}

Written informed consent was obtained from the patient for the publication of this case report. 


\section{Conflict of interest}

The authors declared that there are no potential conflicts of interest with respect to the research, authorship, and/or publication of this article.

\section{Author contributions}

Study concept and design: EF, SA; Acquisition of data: EF, SA; Analysis and interpretation of data: EF, SA; Drafting of the manuscript: EE, SA; Critical revision of the manuscript for important intellectual content: EE, PNK; and Study supervision: PNK.

\section{References}

[1] Moon HK, Kim HS, Heo GM, Shin WG, Kim KH, Jang MK. A case of pedunculated hepatic hemangioma mimicking submucosal tumor of the stomach. Korean J Hepatol 2011;17:66-70.

[2] Chui AK, Vass J, McCaughan GW, Sheil AG. Giant cavernous haemangioma: a rare indication for liver transplantation. Aust $\mathrm{N} \mathrm{Z} \mathrm{J}$ Surg 1996;66:122-4.

[3] Zhang X, Zhou Z. Hepatic hemangioma masquerading as a tumor originating from the stomach. Oncol Lett 2015;9:1406-8.

[4] Liang RJ, Chen CH, Chang YC, Hu RH, Sheu JC. Pedunculated hepatic hemangioma: report of two cases. J Formos Med Assoc 2002;101:437-41.

[5] Vilgrain V, Boulos L, Vullierme MP, Denys A, Terris B, Menu Y. Imaging of atypical hemangiomas of the liver with pathologic correlation. Radiographics 2000;20:379-97. 